Ruibang Luo ${ }^{*}$, Chak-Lim Wong, Yat-Sing Wong, Chi-lan Tang, Chi-Man Liu, Chi-Ming Leung, Tak-Wah Lam*

Department of Computer Science, The University of Hong Kong, Hong Kong, China

${ }^{*}$ Correspondence and requests for materials should be addressed to R. L. (email: rbluo@cs.hku.hk) and T. L. (twlam@cs.hku.hk)

\title{
Abstract
}

14 Single-molecule sequencing technologies have emerged in recent years and revolutionized structural variant calling, complex genome assembly, and epigenetic mark detection.

However, the lack of a highly accurate small variant caller has limited the new technologies

17 from being more widely used. In this study, we present Clair, the successor to Clairvoyante, a program for fast and accurate germline small variant calling, using single molecule sequencing data. For ONT data, Clair achieves the best precision, recall and speed as compared to several competing programs, including Clairvoyante, Longshot and Medaka.

Through studying the missed variants and benchmarking intentionally overfitted models, we found that Clair may be approaching the limit of possible accuracy for germline small variant calling using pileup data and deep neural networks. Clair requires only a conventional CPU 
27 Fast and accurate variant calling is essential for both research and clinical applications of

28 human genome sequencing ${ }^{1,2}$. Algorithms, best practices and benchmarking guidelines have

29 been established for how to use Illumina sequencing to call germline small variants,

30 including single-nucleotide polymorphisms (SNPs) and insertions/deletions (indels) ${ }^{3-6}$. In

31 recent years, single-molecule sequencing (SMS) technologies have emerged for a variety of

32 important applications ${ }^{7}$. These technologies, which are also known as the third-generation

33 sequencing technologies, generate sequencing reads two to three orders of magnitude

34 longer than Illumina reads (10-100kbp versus $100-250 \mathrm{bp})$. The long read length has made

35 the new SMS technologies, including Pacific Biosciences (PacBio) and Oxford Nanopore

36 Technology (ONT), unprecedentedly powerful for resolving complex genome assembly

37 problems and for detecting large structural variants ${ }^{8}$. However, currently available SMS

38 technologies also have a significantly higher base error rate of $3-15 \%{ }^{9}$, making the variant

39 calling methods previously designed for Illumina sequencing inapplicable to SMS

40 technologies. The lack of accurate tools for efficient variant calling has limited SMS

41 technologies from being applied to the many problems that require SNPs and small indels.

43 In our previous work, we developed Clairvoyante ${ }^{10}$, a germline small variant caller for single

44 molecule sequencing data. Clairvoyante does not require sequence assembly and calls

45 variants directly from read alignments. Clairvoyante adopts a deep convolutional neural

46 network, so that by using the truth variants called and orthogonally verified in seven human

47 individuals by the Genome In A Bottle (GIAB) consortium ${ }^{11-13}$, Clairvoyante can be trained

48 for variant calling on any new type of sequencing data without the need to look into its 
49 error profile and build a hand-crafted model. Clairvoyante takes pileup data as input and

50 runs quickly. However, Clairvoyante's design is unable to call multiallelic variants or indels

51 longer than four bases. These defects remain to be solved. Meanwhile, the limit of using

52 pileup data and deep neural networks for variant calling remains to be explored.

53

54 In this study, we present Clair, a fast and accurate system for germline small variant calling

55 using single molecule sequencing data. With an entirely different network architecture and

56 learning tasks (i.e. output components), Clair resolves the multiallelic and long indel variant

57 calling problems that have prevented Clairvoyante from calling all types of small variants.

58 We describe in detail the methods we tried that either worked or did not work for

59 improving Clair's performance. For ONT datasets ${ }^{14}$, our experiments on whole-genome

60 variant calling in GIAB samples show that Clair outperforms Clairvoyante and other variant

61 callers, including Longshot ${ }^{15}$ and Medaka $^{16}$, in terms of precision, recall and speed. For high

62 accuracy reads, including both PacBio CCS (Circular Consensus Sequencing) ${ }^{17}$ and Illumina

63 datasets $^{13}$, DeepVariant ${ }^{18}$ had modestly improved F1-scores over Clair by .11\% to .13\%,

64 although Clair was seven times faster. Looking into the false positive (FP) and false negative

65 (FN) variants of the three sequencing technologies showed that except for variants with

66 insufficient coverage by chance, most of the others could be resolved using complete read

67 alignments instead of pileup data or else could not be resolved at all, even with a manual

68 inspection. 
71 Clair is a four-task, five-layer recurrent neural network with two bi-directional LSTM layers

72 followed by three feedforward layers (Figure 1). Clair takes a BAM file as input to find

73 candidate variants with any minor allele frequencies larger than a threshold (typically

74 between 0.1 and 0.2 ), and then computes a pileup of the candidates and converts the summaries into a tensor. In a tensor, the allelic counts of bases and gaps on both strands of a candidate variant and its 16 flanking bases are encoded into 1,056 integer values. More details and pseudo code are available in the Methods section. As discussed in the Clairvoyante paper, one major unsolved problem was how to support the calling of multiallelic variants (i.e., variants with two alternative alleles). In Clair, the problem is solved by using four new (deep learning) tasks that are entirely different from Clairvoyante. These are:

'Obp', ' $1 b p$ ', ..., '15bp', '>15bp'; and 4) the length of the second indel allele. The 21-genotype probabilistic model can represent all possible genotypes of a diploid sample at the genome position. The length of indels longer than $15 \mathrm{bp}$ cannot be directly inferred from the third and fourth tasks, so Clair includes an additional step that re-scans the alignments. More details on each of these steps can be found in the Methods section. The four tasks make

90 their own decisions and are designed to cross-validate each other. For example, task two is

91 a coarse-grained version of task one and can veto the decision made by task one. Tasks 
92 three and four should indicate Obp indel length if an SNP variant is decided by task one.

93 More details on how the four tasks make a joint decision are available in the Methods

94 section. We used the 'focal loss' deep-learning technique to solve the problem of

95 unbalanced variant types in training data. We used the 'cyclical learning rate' deep learning

96 technique to achieve the maximum possible variant calling performance and speed up the

97 training process to be able to handle larger training datasets. To improve Clair's

98 performance at lower sequencing coverages, we augmented the training data with 10

99 subsampled coverages of each dataset. The parameters of these three new techniques are

100 in the Methods section.

101

102 Clair has $2,377,818$ parameters, which is $45.7 \%$ more than Clairvoyante $(1,631,496$

parameters) but only one tenth as many as DeepVariant $(23,885,392$ parameters). In terms

of variant calling speed, Clair takes about 30 minutes, 1.5 hour, and 5 hours for a 50 -fold

coverage WGS sample using Illumina, PacBio CCS and ONT data, respectively, using 24 CPU

cores. In our experiments, Clair was $10-20 \%$ slower than Clairvoyante, but significantly

107 faster than DeepVariant, Longshot and Medaka.

109 The Methods section includes a description of procedures to augment the training data or

110 improve Clair's network architecture that we tested but that did not improve precision and

111 recall of variant calling. Developers working on further improving Clair's performance can

112 save time by avoiding the same methods, or the same settings in a method. 
115 ONT datasets are currently available for two GIAB samples, HG001 and HG002. The HG001

116 rel6 dataset generated by the Nanopore WGS Consortium ${ }^{14}$ contains approximately 44.3-

117 fold coverage of human genome (the dataset is also referred to as 1:44x, where ' 1 ' means

118 the sample suffix and ' $44 x^{\prime}$ ' means the coverage). The rel6 dataset was base-called with

119 Guppy 2.3.8, using the HAC (High-ACcuracy) model. In addition to the rel6 dataset, we

120 obtained a separate 124.1-fold coverage dataset for HG001 (1:124x) directly from Oxford

121 Nanopore (Philipp Rescheneder, personal communication). That dataset was base-called

122 with Guppy 2.2.3 using the Flip-Flop model. In some experiments, we combined 1:44x and

$1231: 124 x$ to form a new dataset 1:168x to maximize the coverage. For HG002, we used a

124 dataset with 64-fold coverage (2:64x) from the GIAB consortium, which was base-called

125 with Guppy 2.3.5 using the Flip-Flop model. The links to the datasets are available in the

126 Supplementary Notes. The details about "the GIAB truth variant datasets", "removing

127 GA4GH (The Global Alliance for Genomics and Health) low-complexity regions ${ }^{6}$ from

128 benchmarking", and "the benchmarking methods and metrics" are available in "Methods -

129 Benchmarking".

131 Figure 2 shows the precision and recall of Clair and other variant callers on SNPs and indels

132 in multiple experiments with ONT data. Supplementary Table 1 contains more details,

133 including precision, recall and F1-score in five categories, including overall, SNP, indel,

134 insertion, and deletion. Our results show that Clair not only outperformed other variant

135 callers, including Clairvoyante, Longshot, and Medaka, but also ran much faster. Using

$1361: 168 x \mid 2: 64 x$ (i.e., test variant calling using HG002 with 64-fold coverage against a model

137 trained using HG001 with 168-fold coverage) as Clair's primary result, Clair achieved $98.36 \%$ 
precision, $96.46 \%$ recall, and $97.40 \%$ F1-score overall performance. In terms of SNPs, the

139 three metrics were $99.29 \%, 97.78 \%$ and $98.53 \%$, respectively. For indels, they were

140 somewhat lower at $81.15 \%, 73.88 \%$, and $77.34 \%$. Clair significantly outperformed its

141 predecessor Clairvoyante on both SNP and indel calling (overall F1-score $97.40 \%$ versus

142 93.45\%). Clair had a slightly higher F1-score on SNPs than Longshot (98.53\% versus 98.41\%),

143 but Longshot detects only SNPs, and Clair ran five times faster than Longshot (320 versus

1441,797 minutes). Clair had a better performance than Medaka (overall F1-score $97.40 \%$

that we didn't benchmark Nanopolish ${ }^{19}$, which is also capable of variant calling on ONT data,

because it also requires raw signals as input, which are not publicly available for HG002.

We ran further experiments to answer five additional questions about Clair, as follows.

Is the Clair model reference-genome specific? In our experiments, performance did not

depend on whether we used GRCh37 or GRCh38. The performance of 1:168x|2:64x and

1:168x | 2:64x(b37) was similar; the latter experiment tested HG002 GRCh37 read alignments

on a model trained using HG001 GRCh38 read alignments. Actually, 1:168x |2:64x(b37)

performed slightly better than 1:168x|2:64x, with a $0.18 \%$ better F1-score on SNPs, and

$1.4 \%$ on indels.

Does higher coverage in the test sample helps improve variant calling performance? Yes,

159 but improvement seems to asymptote at $\sim 60$-fold coverage. In a comparison of 
$162(+8.47 \%)$. Further increasing the coverage in the test sample will note significantly increase

163 the variant calling performance as we discuss below.

Does higher coverage for model training help improve variant calling performance? Yes,

but it depends on the coverage of the test sample. In a comparison of $1: 124 x \mid 2: 64 x$ to

$98.01 \%$ to $98.54 \%(+0.53 \%)$, and the indel from $75.78 \%$ to $78.44 \%(+2.66 \%)$. In a comparison

170 from $97.51 \%$ to $97.40 \%$ overall. One possible reason is that the lower coverage test sample

Does multiple subsampled coverage for model training improved variant calling

$17975.78 \%$ to $78.44 \%(+2.86 \%)$. The results show that even without sufficient coverage for performance significantly.

183 What is the upper bound on performance?

184 To determine Clair's performance cap using the current ONT data, we intentionally 
though Clair is designed with multiple generalization techniques, including 'dropout' and 'L2

187 regularization', exposing the test samples to model training is a biased evaluation, and if a

true variant is not called even after this biased training, this suggests the input signal is

simply too weak. The two tests we did were $1: 168 x+2: 64 x \mid 2: 64 x$ and $1: 168 x+2: 64 x \mid 1: 168 x$.

Although the test sample coverage in the first test was much lower than that in the second

(64-fold against 168-fold), their performance was similar, with the overall F1-score at

$97.77 \%$ and $97.82 \%$, SNP at $98.75 \%$ and $98.77 \%$, and indel at $79.92 \%$ and $81.37 \%$. The

biased test 1:168x+2:64x|2:64x did not significantly outperform 1:168x|2:64x; the overall

F1-score increased from $97.40 \%$ to $97.77 \%$ (+0.33\%), SNP from $98.53 \%$ to $98.75 \%(+0.22 \%)$,

that the performance of using Clair on the current ONT data was capped at about $97.8 \%$ F1-

that provides a lower base error rate can raise the upper bound of Clair's variant calling

performance in the Discussion section below.

We analyzed and categorized the FP and FN results of Clair on ONT data. We randomly

extracted 100 FPs and 100 FNs from the 1:168x|2:64x experiment. Figure 3 shows a

207 other alleles in the read alignments (including the correct one), and the incorrect allele has a 
worth mentioning that these repetitive regions are $\leq 10 \mathrm{bp}$ because we removed all GA4GH

211 low-complexity regions longer than $10 \mathrm{bp}$ from benchmarking. It may not be possible to

212 perfectly resolve these three categories for FP variants using pileup data for variant calling,

213 although complete read alignments might help to provide better precision. Three out of 100

214 FPs had "Incorrect insertion bases", while two out of 100 were categorized as "Overlapping

215 insertions", which means that the alleles of two consecutive insertions overlapped each

216 other in an input tensor; thus, the correct allele cannot be resolved for both insertions.

217 These two categories of errors can be resolved using the '--pysam_for_all_indel' option in

218 Clair, but this slows down Clair for ONT data by a factor of up to ten times. Other errors,

219 including "Incorrect indel length" and "Incorrect zygosity", are errors made by Clair's neural

220 network. In the $100 \mathrm{FNs}$, the three major categories are "Correct allele with $\mathrm{AF}<0.25$ "

221 (54/100), "Homopolymer" (18/100), and "Tandem repeat" (7/100). "Correct allele with

$222 \mathrm{AF}<0.25$ " means that at the location of the missed (FN) variant, the signal of the correct

223 allele is rather weak, with allele frequency lower than $25 \%$. One FN categorized as "More

224 than two possible alternative alleles" is an error due to an alignment error in segmental

225 duplications, in which more than two alternative alleles seem correct.

228 In early $2019^{17}$, PacBio developed a protocol based on single-molecule, circular consensus

229 sequencing (CCS) to generate highly accurate (99.8\%) long reads averaging as much as

$23013.5 \mathrm{~kb}$. PacBio published CCS datasets for HG001 (in this section also referred to as 1:30x; 1

231 as the sample suffix and 30x means 30-fold coverage), HG002 (2:33x) and HG005 (5:33x). All

232 three samples are involved in model training. To demonstrate a possible overfitting 
233 phenomenon on deep learning based variant callers, both HG002 and HG005 are used in

234 benchmarking.

235

236 Supplementary Table 3 shows the results of Clair and three other variant callers:

237 Clairvoyante, Longshot, and DeepVariant. Testing on HG002, DeepVariant performed the

238 best, with an overall F1-score of $99.96 \%$, SNP of $99.97 \%$, and indel of $99.92 \%$. The primary

239 result of Clair 1:30x+5:33x|2:33x had an overall F1-score of $99.83 \%$, which was $0.13 \%$ lower

240 than DeepVariant, but outperformed both Clairvoyante and Longshot. On SNP,

$2411: 30 x+5: 33 x \mid 2: 33 x$ had an F1-score of $99.88 \%$, which was $0.09 \%$ lower than DeepVariant,

$242 \quad 0.43 \%$ higher than Longshot, and $0.17 \%$ higher than Clairvoyante. On indel,

$2431: 30 x+5: 33 x \mid 2: 33 x$ had an F1-score at $99.07 \%$, which was $0.85 \%$ lower than DeepVariant,

244 but $19.17 \%$ higher than Clairvoyante, showing that the new methods applied to Clair have

245 effective solved the indel-calling problem in Clairvoyante. In terms of speed, Clair (147

246 minutes) is slightly faster than Longshot (206 minutes), and about seven times faster than

247 DeepVariant (1,072 minutes). We also tested HG005. Interestingly, while Clair, Clairvoyante,

248 and Longshot all performed better on HG005 than HG002, DeepVariant performed worse.

249 Comparing 1:30x|2:33x to 1:30x|5:33x, Clair's overall F1-score increased from 99.77\% to

$25099.80 \%$. Clairvoyante's overall F1-score increased from $98.61 \%$ to $98.70 \%$. Longshot's SNP

251 F1-score increased from $99.45 \%$ to $99.46 \%$. The performance of the three callers verifies the

252 quality of the HG005 dataset. However, DeepVariant's F1-score dropped from 99.96\% to

$25399.92 \%$, the SNP F1-score decreased from 99.97\% to 99.93\%, and the indel F1-score

254 dropped most significantly, from $99.92 \%$ to $99.78 \%$. The most probable reason is that,

255 DeepVariant's current PacBio CCS model was trained completely using $\mathrm{HGOO2}^{20}$. We suggest

256 using DeepVariant's result on HG005 as its real performance on PacBio CCS data. The biased 
test $1: 30 x+2: 33 x+5: 33 x \mid 2: 33 x$ found the performance cap of Clair at $99.88 \%$ on SNP, which

was the same as $1: 30 x+5: 33 x \mid 2: 33 x$, and $99.28 \%$ on indel, which was $0.21 \%$ higher than

training was only $33 \mathrm{x}$, we expect to fill the performance gap on indel calling by using higher

DeepVariant uses complete read alignments that contain information at a per-read level.

264 This is also a reason DeepVariant runs slower than Clair. We discuss the possibility of

265 improving Clair to use complete read alignments without slowing down performance in the 266 Discussion section below.

\section{Performance on Illumina}

269 Approximately 300x coverage in 148-bp Illumina paired-end read data is available for five

270 GIAB samples, including HG001, HG002, HG003, HG004 and HG005 ${ }^{11}$. We used HG001,

$271 \mathrm{HG003}, \mathrm{HGO04,} \mathrm{HG005} \mathrm{for} \mathrm{model} \mathrm{training,} \mathrm{and} \mathrm{HG002} \mathrm{for} \mathrm{benchmarking.} \mathrm{To} \mathrm{resemble} \mathrm{the}$

272 typical coverage in whole genome sequencing, we used full coverage of HG001 (306-fold)

and HGOO5 (352-fold), but down-sampled HG002, HG003 and HG004 to 52-, 57-, and 66-

274 fold.

Supplementary Table 4 shows the results of Clair and DeepVariant. DeepVariant performed 
281 DeepVariant's (99.48\% versus 99.90\%). In terms of speed, Clair was about seven times faster

282 than DeepVariant (77 versus 537 minutes). The biased test

$2831: 306 x+2: 52 x+3: 57 x+4: 66 x+5: 352 x \mid 2: 52 x$ found the performance cap of Clair to be $99.87 \%$

284 for SNPs, which was $0.02 \%$ higher than the primary result, but $0.07 \%$ lower than that of

285 DeepVariant, and $99.57 \%$ for indels, which was $0.09 \%$ higher than the primary result, but

$2860.33 \%$ lower than that of DeepVariant. Similar to the ONT and PacBio CCS experiments, we

287 expect to fill in the performance gap through partially making use of complete read

288 alignments, as discussed in the Discussion section.

In this paper we present Clair, a germline small variant caller for single molecule sequencing data. The name Clair means 'clear' in French, echoing its predecessor, named Clairvoyante, meaning 'clear seeing'. Clair adds new methods to solve problems that Clairvoyante had trouble with, including multiallelic variant calling and long indel calling. In our experiments on ONT data, Clair outperformed all existing tools in terms of precision, recall and speed. On PacBio CCS and Illumina data, Clair performed slightly worse than DeepVariant, but ran about an order of magnitude faster. Looking closer at the FP and FN variants shows that

Clair is approaching the limit on how accurately it can call variants using pileup data. Some of the erroneous variant calls can be corrected using complete read alignments instead of pileup data. However, dealing with complete read alignments requires a more powerful neural network design with much greater computational demands. In the future, we will 
304 The quality and sufficiency of training data is key to the performance of Clair, as well as

305 other deep learning based variant callers, such as DeepVariant. To train a model for

306 production purposes, we used five samples (HG001 to 5) for Illumina data, but only two

307 samples (HG001 and HG002) for ONT, due to the limited availability of public high-coverage

308 whole genome sequencing datasets for the GIAB samples. ONT sequencing of the other

309 GIAB samples is ongoing, and more data will be available in the near future. With additional

310 datasets, we expect to see even higher performance in Clair on ONT data.

312 On ONT data, although Clair performed the best, its indel calling precision and recall were

313 only about $80 \%$, even excluding GA4GH low-complexity regions, which leaves substantial

314 room for improvement. While the precision can be further improved by considering

315 complete read alignments, the recall is bounded by input and can be improved only with a

316 lower read-level base-calling error rate. Future improvements in ONT technology offer the

317 possibility of reducing the error rate to $2-3 \%$, which in turn should improve Clair's ability to

318 detect indels in these data.

320 The GIAB datasets we used for model training have moderate whole-genome sequencing coverage. Although we can use samples with very high coverage (over 300 -fold, which is sometimes seen in amplicon sequenced data) with Clair for variant calling, such samples might show degraded performance because very high coverage variants were not adequately observed in model training. To solve this problem, we propose two methods.

325 One method is to do transfer learning using a trained model on additional datasets with very high coverage. Clair supports transfer learning and can be applied to additional

327 datasets instantly. Another method is an ensemble method, which generates multiple 
copies of randomly subsampled read alignments at a candidate variant for Clair to call

329 variant. A majority vote or a decision tree can be used to make the final decision, using the

330 results of each copy.

332 A limitation of Clair is that it cannot be applied to polyploid species, which are inconsistent

333 with its neural network design. For the same reasons, Clair is not applicable to somatic

334 variant calling, where a single sample might hold multiple distinct populations of cells. Our

335 next steps include extending Clair to support polyploid species and somatic variant calling.

340 For a truth variant for training or a candidate variant for calling, the read alignments that

341 overlap or are adjacent to the variant are summarized (i.e. pile-up data) into a three-

342 dimensional tensor of shape 33 by 8 by 4, comprising 1056 integer numbers. The three

343 dimensions correspond to the position, the count of four possible bases from two different

344 strands, and four different ways of counting. In the first dimension, 33 positions include the

345 starting position of a variant at the center and 16 flanking bases on both sides. The second

346 dimension corresponds to the count of 'A+', 'A-', 'C+', 'C-', 'G+', 'G-', 'T+' or 'T-', with the

347 symbols +/- denoting the count from the forward/reverse strand. The third dimension

348 replicates the first two dimensions with four different ways of counting to highlight 1) the

349 allelic count of the reference allele, 2) insertions, 3) deletions and 4) single nucleotide

350 alternative alleles. "Supplementary Note - Pseudocode for generating the input tensor" 
351 shows the pseudo code of the exact algorithm of how the input tensor is generated.

352 Supplementary Figure 1 demonstrates how the tensors are look like for ONT data at a random 'non-variant', a 'SNP', an 'Insertion', and a 'Deletion'.

\section{Output}

356 The output of Clair has four tasks (a.k.a. four output components, in total 90 probabilities),

357 including 1) the 21-genotype probabilistic model (21 probabilities); 2 ) zygosity (3

358 probabilities); 3 ) the length of the first indel allele (33 probabilities); and 4) the length of the second indel allele (33 probabilities). One of the breakthroughs in Clair is the invention of the 21-genotype probabilistic model. It comprises all of the possible genotypes of a diploid sample at a genome position, including 'AA', 'AC', 'AG', 'AT', 'CC', 'CG', 'CT', 'GG', 'GT', 'TT', 'Al', 'Cl', 'GI', 'TI', 'AD', 'CD', 'GD', 'TD', 'II', 'DD', and 'ID', where 'A', 'C', 'G', 'T', 'I' (insertion) and ' $D$ ' (deletion) denote the six possible alleles. The new model covers variants with two alternative alleles, which could not be called in Clairvoyante. The zygosity task outputs the probability of the input being 1 ) a homozygous reference $(0 / 0) ; 2$ ) heterozygous with 1 or 2 partially redundant to the 21-genotype task, but it makes decisions independently, and it crosschecks the decision made by the 21-genotype task. Tasks three and four have the same design. They output the length of up to two indel alleles. Each task outputs 33 probabilities, including the likelihood of 1) more than $15 b p$ deleted (<-15bp); 2 ) any number between $15 \mathrm{bp}$ and $15 \mathrm{bp}$, including $0 \mathrm{bp}$, and; 3) more than 15bp inserted (>15bp). In training, the indel allele with a smaller number is set as the first indel allele. For example, for a heterozygous $1 b p$ deletion, the first indel allele is set as $-1 b p$, the second as $0 b p(-1 b p / 0 b p)$.

374 For a heterozygous $1 b p$ insertion, $0 b p / 1 b p$ is set. This design makes the non-0bp training 
variants for both tasks balanced. For a heterozygous indel with two alternative alleles, say, one $-2 b p$ and one $5 b p,-2 b p / 5 b p$ are set. For a homozygous indel, two indel alleles are set to the same value. For indels longer than $15 \mathrm{bp}$, the exact length is determined using an additional step (Supplementary Note - New methods used in Clair - Dealing with indels longer than 15bp). The output of the two indel allele tasks are also used for crosschecking with the 21-genotype task, with Obp supporting an SNP allele, and non-Obp supporting an indel allele. More details about how the four tasks crosscheck each other to come up with a result coherently are in "Method - New methods used in Clair - Determining the most probable variant type using the four tasks of Clair".

386 Clair has been fully revamped while a few basic deep-learning techniques in Clairvoyante

392 For each candidate variant, Clair directly outputs the length of up to two alternative indel 
the second-highest allelic count for 'II'; 3) the deletions with highest and/or the second-

highest allelic count for 'DD', or; 4) both the insertion and deletion with the highest allelic

401

count for 'ID'. The additional step is slow, but it is required only for indels longer than $15 \mathrm{bp}$.

We investigated HG001 and found 570,367 indels in its truth variant set; only 10,672

403

(1.87\%) were $>15 \mathrm{bp}$. In our experiments, we found the slowdown was acceptable. Users can

417 into 10 categories: 1) a homozygous reference allele; 2 ) a homozygous 1 SNP allele; 3) a

418 heterozygous 1 SNP allele, or heterozygous 2 SNP alleles; 4) a homozygous 1 insertion allele;

419 5) a heterozygous 1 insertion allele, or heterozygous 1 SNP and 1 insertion alleles; 6 )

420 heterozygous 2 insertion alleles; 7) a homozygous 1 deletion allele; 8) a heterozygous 1

421 deletion allele, or heterozygous 1 SNP and 1 deletion alleles; 9) heterozygous 2 deletion 
42310 categories is calculated for each candidate variant, and the category with the largest

424 likelihood value is chosen (Pseudocode in "Supplementary Note - Pseudo code for

425 determining the most probable variant type"). The variant quality is calculated as the square

426 of the Phred score of the distance between the largest and the second-largest likelihood

427 values.

428

429

Cyclical learning rate

430 The "initial learning rate" and "how the learning rate decays" are two critical

431 hyperparameters in training a deep neural network model. A model might be stuck at a local

432 optimum (i.e. unable to achieve the best precision and recall) if the initial learning rate is

433 too large, or the decay is too fast. But a large initial learning rate, and a slow decay rate

434 make the training process either unstable or take too long to finish. So in common practice,

435 a tediously long grid search that is very costly is needed to find the best hyperparameters.

436 Furthermore, through a grid search, we found that different sequencing technologies differ

437 in their best hyperparameters. This problem makes model training too complicated and

438 largely impedes Clair from being applied to new datasets and sequencing technologies. To

439 solve the problem, we implemented Cyclical Learning Rate (CLR) ${ }^{22}$ in Clair. CLR is a new deep

440 learning technique that eliminates the need to find the best values of the two

441 hyperparameters. CLR gives a way to schedule the learning rate in an efficient way during

442 training, by cyclically varying between a lower and higher threshold. Following the CLR

443 paper, we determined the higher threshold to be 0.03 and the lower threshold to be 0.0001 .

444 The two thresholds worked well on the training variants of all three sequencing

445 technologies (Illumina, PacBio CCS and ONT). In terms of which CLR scheduler to use, we

446 chose the triangular schedule with exponential decay. In our experiments, on PacBio CCS 
447 and Illumina datasets, CLR decreased model training time by about 1-3 times, while often

448 outperforming the three-step decay method introduced in Clairvoyante for both precision

449 and recall. However, on ONT datasets, CLR has a lower, but almost negligible, performance

450 than the three-step decay. We provide both CLR and three-step decay options in Clair. To

451 train a model for production, we suggest users try both options and choose the best

452 through benchmarking. In our results, we used CLR for PacBio CCS and Illumina datasets,

453 and the three-step decay method for ONT datasets.

456 Our training data uses the truth variants from the GIAB consortium and is unbalanced in

457 terms of variant type. For example, the number of heterozygous variants is nearly twice that

458 of the homozygous variants. SNPs are about five times more numerous than indels. Worst

459 of all, only $\sim 1.1 \%(39,898$ of $3,619,471$ in HG001) of variants have two or more alternative

460 alleles. And among them, only $884(\sim 0.024 \%)$ are multiallelic SNPs. This problem leads to

461 degenerate models, as the numerous easy variants contribute no useful learning signals and

462 overwhelm training. In our practice, if we leave the problem unaddressed, we observe a

463 significant drop in recall for the underrepresented variant types. For multiallelic SNPs, the

464 recall dropped to zero. To solve this problem, we used the "Focal loss" technique ${ }^{23}$, which

465 applies a modulating term to the cross-entropy loss in Clair's output to focus training on

466 underrepresented hard variants and down-weight the numerous easy variants. Focal loss

467 calculates the loss as $\left(1-p_{t}\right)^{\gamma} \times \alpha_{t} \times-\log \left(p_{t}\right)$, where $p_{t}=p, \alpha_{t}=\alpha$, if the prediction

468 matches the truth, or $p_{t}=(1-p), \alpha_{t}=(1-\alpha)$ otherwise. In addition to the traditional

469 cross entropy loss, focal loss uses two more parameters: $\gamma$ (the focusing parameter) to

470 differentiate easy/hard training examples, and $\alpha$ (the balancing parameter) to balance the 
471 importance of positive/negative training examples. We determined $\gamma=2$ and $\alpha=0.25$

472 work best for the GIAB truth variants with a 1:2 ratio of truth variant and non-variant. The

473 use of focal loss significantly increases the performance of underrepresented variant types.

474 It also allows us to be more lenient on variant type balance when augmenting the training

475 data.

476

477 Training data augmentation using subsampled coverage

478 Lower coverage usually leads to lower precision and recall in variant calling. To train Clair to

479 achieve better performance on variants with lower coverages, we subsampled each dataset

480 into four or nine additional datasets with lower coverages. The subsampling factors $f$ are

481 determined as $(\sqrt[h]{4 \div c})^{n}$, where $c$ is full coverage of each sample, 4 is the minimal

482 coverage, $h$ is either 4 or 9 , and $n$ is from 1 to $h$. Using HG002 as an example, its full

483 coverage is 63.68-fold, and the nine subsampled coverages are 46.82-, 34.43-, 25.31-,

484 18.61-, 13.69-, 10.06-, 7.40-, 5.44- and 4.00-fold. If variant samples were lower than $4 x$ after subsampling, we removed them from training. We used the command "samtools view -s $f$ " to generate a subsampled BAM. A different seed counting from zero for random number generation was set for each coverage. The use of subsampled coverages improved the recall on indel significantly.

In this section we discuss methods we tested that had no effect on Clair's performance. For avoided or revised. 
496 Intuitively, a larger input tensor with more flanking bases provides additional information

497 on the surrounding read alignments, which might lead to better precision and recall. Our

498 experiments show that extending the input tensor from 33bp (16bp flanking bases) to 49bp

499 (24bp flanking bases) and 65bp (32bp flanking bases) slows down Clair by $5.4 \%$ and $12.6 \%$,

500 respectively. But the improvement was negligible in terms of precision or recall with both

501 SNP and indel.

502

503

Using non-variants adjacent to true variants as negative samples for model training

504 Clair, by default, uses a ratio of 1:2 on true variants and non-variants for model training, and

the non-variants are randomly selected from the genome, except for the positions with a

true variant or insufficient coverage. We experimented using non-variants adjacent to true

507 variants (we tried $\pm 2 \mathrm{bp}, \pm 8 \mathrm{bp}$ and $\pm 16 \mathrm{bp}$ ) as negative samples for model training and

adjusted the ratio to 1:1:1 on true variants adjacent non-variants and random non-variants.

509 We used adjacent non-variants for training because their input is true variant alike, but a

510 few bases shifted. The hypothesis was that using them as adversarial training samples

511 against the true variants might improve Clair's performance at high density variants and

512 alignment errors. However, our experiments show that the method decreased recall slightly

513 on both SNP and indel.

516 The GIAB HG001 truth variant dataset includes 3,619,471 truth variants passing all criteria

517 (with the 'PASS' tag), and 2,264,796 variants failing one or more criteria. The criteria details

518 were explained by Zook et al. in $2019^{13}$. Among the failed variants, 310,113 had the 
519 'allfilteredbutagree' tag, which means at the same position, the variants called in all the

520 supporting datasets agreed with each other, even though none of them were in the callable

521 regions, in which a range of coverage and minimum alignment quality are met. These

522 variants are considered less confident than those passing all criteria, but might still

523 contribute to training a better model because while a deep neural network can tolerate

524 moderate errors in training data, if any new patterns are provided in additional data, it will

525 be learned by the model and, in turn, improve the performance. We experimented adding

526 the variants with the 'allfilteredbutagree' tag to training. However, our results show that the

527 recall went down significantly on SNP, and the precision went down significantly on indel.

530 Variant calling in homopolymer sequences is usually more challenging, and the problem is

531 even worse in SMS technologies since the length of homopolymers is usually

532 underestimated. At longer homopolymers, the signals are usually too discordant, so it is

533 common for humans to make mistakes with them. From the feature engineering point of

534 view, variants in homopolymer sequences are confusing and less informative, and might

535 lead to a degenerate model. We tested model training without variants at homopolymer

536 sequences longer than $5 \mathrm{bp}$. Our results show that both precision and recall degrade

537 significantly if homopolymer variants are not used in model training.

539 Benchmarking

\section{The GIAB truth variant datasets}

541 We used the GIAB version 3.3.2 datasets as our truth variants. Depending on the availability

542 of deep sequencing data, our ONT experiments used samples HG001 or HG001+HG002 for 
model training, our PacBio CCS experiments used HG001 or HG001+HG005, and our Illumina

544 experiments used $\mathrm{HG001}$ or $\mathrm{HG001+HG003+HG004+HG005}$. For benchmarking, ONT, PacBio

545 CCS and Illumina experiments have used HG002, HG005, and HG002, respectively. The links

546 to the truth variants and high-confidence regions are available in "Methods - Data sources -

547 Truth variants". Depending on the reference genome used in the already available read

548 alignments, we used GRCh38 for our ONT and Illumina experiments, and GRCh37 for our

549 PacBio CCS experiments. The links to the reference genomes we used are available in

550 "Methods - Data sources - Reference genomes"

Removing GA4GH low-complexity regions from benchmarking

553 Krusche et al. ${ }^{6}$ from the GA4GH benchmarking team and the GIAB consortium published the

554 low-complexity regions, including homopolymers, STRs, VNTRs, and other repetitive

555 sequences for stratifying variants in their paper titled "Best practices for benchmarking

556 germline small-variant calls in human genomes". In the low-complexity regions larger than

557 10bp, ONT's performance degraded significantly (precision $-11.41 \%$, recall $-55.33 \%$ ), while

558 that of PacBio CCS and Illumina dropped only $0.99-1.67 \%$ in precision and recall

559 (Supplementary Table 5). Thus, when computing variant calling using ONT, we suggest

560 removing the variants called in the low-complexity regions. In our benchmarks for all

561 datasets, in addition to using the high-confidence regions of each sample provided by GIAB,

562 we removed the low-complexity regions. The procedures are available in "Supplementary

563 Note - Commands - Remove GA4GH low complexity regions from GIAB's high-confidence

564 regions". There was retention of 92.61-93.47\% high-confidence regions in GRCh38, and

$56594.40-95.05 \%$ in GRCh37 of the five samples HG001 to 5 after removing the low-complexity

566 regions (Supplementary Table 8). 


\section{Benchmarking methods and metrics}

569 Clair trains a model either for 30 epochs, using the Cyclical Learning Rate (used for PacBio

570 CCS and Illumina datasets), or by decaying the learning rate three times (by one tenth each

571 time) until the validation losses converge (used for ONT datasets). While the performance of

572 last few epochs are generally similar, the best-performing one will be chosen for

573 benchmarking. We did not run replications of model training because choosing from the

574 best epoch actually resembles the process of having multiple replications. In ONT and

575 Illumina experiments, the GRCh38 reference genome was used, while in PacBio CCS

576 experiments, GRCh37 was used. For each variant calling experiment, we used the

577 submodule vcfeval in RTG Tools ${ }^{24}$ version 3.9 to generate three metrics, 'Precision', 'Recall',

578 and 'F1-score', for five categories of variants: 'Overall', 'SNP', 'Indel', 'Insertion', and

579 'Deletion'. All time consumptions were gauged on two 12-core Intel Xeon Silver 4116 (in

580 total 24 cores), with 12 concurrent Clair processes, each with 4 Tensorflow threads. As Clair

581 has some serial steps that use only one thread, we observed our setting sufficient to

582 maximize the utilization of all 24 cores. For other variant callers, including DeepVariant,

583 Longshot and Medaka, options were to set to use all 24 cores for the best speed.

586 Clair requires Python3, Pypy3 and Tensorflow. Variant calling using Clair requires only a

587 CPU. For a typical 30-fold human WGS sample, Clair takes about an hour for Illumina data

588 and PacBio CCS data, and five hours on ONT data, using two 12-core Intel Xeon Silver 4116

589 processors. Memory consumption depends on both input data and concurrency. ONT data

590 has a higher memory footprint than Illumina and PacBio CSS, while Clair is capped at 7GB 
per process (helper scripts at 4.5GB and Tensorflow at 2.5GB). Model training requires a

592 high-end GPU; we used the Nvidia Titan RTX 24GB in our experiment. Using Clair's default

parameters, generating 1 million training samples takes about 38 seconds. For example, the

594 Illumina model with four samples (HG001, 3, 4, 5) and 30 coverages in total (10 for 1 and 5,

5955 for 2 and 3 ) has $284,367,735$ training samples and takes about 11,000 seconds per epoch.

596 In comparison, the Nvidia RTX $2080 \mathrm{Ti} 11 \mathrm{~GB}$ is about $15 \%$ slower, and the Nvidia GTX $1080 \mathrm{Ti}$

$59711 \mathrm{~GB}$ is about $35 \%$ slower.

598

599

Code availability

600 Clair is open source, available at https://github.com/HKU-BAL/Clair.

601

603 The authors declare that all data supporting the findings of this study are available at the

604 links in the paper and its supplementary information files.

\section{Acknowledgements}

606 We thank Steven Salzberg, Mike Schatz, and Fritz Sedlazeck for their valuable comments. R.

607 L. was supported by the ECS (Grant No. 27204518) of the HKSAR government, and the URC

608 fund at HKU. T. L., C. W., Y. W., C. T., C. Li. and C. Le. were supported by the ITF (Grant No.

609 ITF/331/17FP) from the Innovation and Technology Commission, HKSAR government.

\section{Author contributions}

611 R. L. and T. L. conceived the study. R. L, C. W., Y. W., C. T., C. Li. and C. Le. analyzed the data

612 and wrote the paper. 
bioRxiv preprint doi: https://doi.org/10.1101/865782; this version posted December 16, 2019. The copyright holder for this preprint (which was not certified by peer review) is the author/funder, who has granted bioRxiv a license to display the preprint in perpetuity. It is made available under aCC-BY-NC-ND 4.0 International license.

\section{Competing interests}

614 The authors declare no competing interests

615 
6171 Goodwin, S., McPherson, J. D. \& McCombie, W. R. Coming of age: ten years of next-

618 generation sequencing technologies. Nat Rev Genet 17, 333-351,

619 doi:10.1038/nrg.2016.49 (2016).

6202 Ashley, E. A. Towards precision medicine. Nat Rev Genet 17, 507-522,

621 doi:10.1038/nrg.2016.86 (2016).

$6223 \mathrm{Li}, \mathrm{H}$. Toward better understanding of artifacts in variant calling from high-coverage

623 samples. Bioinformatics 30, 2843-2851, doi:10.1093/bioinformatics/btu356 (2014).

625 Luo, R., Schatz, M. C. \& Salzberg, S. L. 16GT: a fast and sensitive variant caller using a 16-genotype probabilistic model. GigaScience (2017).

6265 Van der Auwera, G. A. et al. From FastQ data to high confidence variant calls: the

627

628

629 Genome Analysis Toolkit best practices pipeline. Curr Protoc Bioinformatics 43, 1110 11-33, doi:10.1002/0471250953.bi1110s43 (2013).

630

631

632 Krusche, P. et al. Best practices for benchmarking germline small-variant calls in human genomes. Nat Biotechnol 37, 555-560, doi:10.1038/s41587-019-0054-x (2019).

7 The long view on sequencing. Nat Biotechnol 36, 287, doi:10.1038/nbt.4125 (2018). Sedlazeck, F. J., Lee, H., Darby, C. A. \& Schatz, M. C. Piercing the dark matter: bioinformatics of long-range sequencing and mapping. Nat Rev Genet, doi:10.1038/s41576-018-0003-4 (2018).

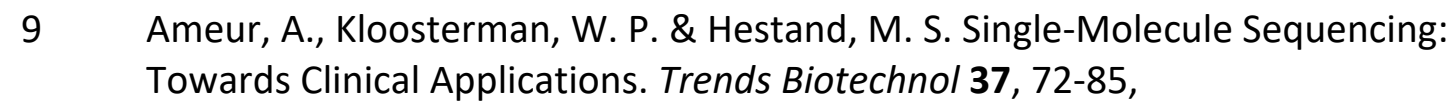
doi:10.1016/j.tibtech.2018.07.013 (2019).

10 Luo, R., Sedlazeck, F. J., Lam, T. W. \& Schatz, M. C. A multi-task convolutional deep neural network for variant calling in single molecule sequencing. Nat Commun 10, 998, doi:10.1038/s41467-019-09025-z (2019).

11 Zook, J. M. et al. Extensive sequencing of seven human genomes to characterize benchmark reference materials. Sci Data 3, 160025, doi:10.1038/sdata.2016.25 (2016). doi:10.1038/nbt.2835 (2014).

13 Zook, J. M. et al. An open resource for accurately benchmarking small variant and reference calls. Nature Biotechnology 37, 561-566, doi:10.1038/s41587-019-0074-6 (2019).

14 Jain, M. et al. Nanopore sequencing and assembly of a human genome with ultralong reads. Nat Biotechnol 36, 338-345, doi:10.1038/nbt.4060 (2018).

15 Edge, P. \& Bansal, V. Longshot enables accurate variant calling in diploid genomes from single-molecule long read sequencing. Nat Commun 10, 4660, doi:10.1038/s41467-019-12493-y (2019).

16 medaka: Sequence correction provided by ONT Research. https://github.com/nanoporetech/medaka, accessed Nov 172019. 
66118 Poplin, R. et al. A universal SNP and small-indel variant caller using deep neural Simpson, J. T. et al. Detecting DNA cytosine methylation using nanopore sequencing.

665 Nature methods 14, 407 (2017).

666

20 Poplin, R. et al. DeepVariant training data.

669 https://github.com/google/deepvariant/blob/r0.9/docs/deepvariant-details-

670 training-data.md, accessed Nov 222019.

21 Li, H. et al. The Sequence Alignment/Map format and SAMtools. Bioinformatics 25,

671 2078-2079, doi:10.1093/bioinformatics/btp352 (2009).

672 Smith, L. N. in 2017 IEEE Winter Conference on Applications of Computer Vision (WACV). 464-472 (IEEE). international conference on computer vision. 2980-2988.

675 


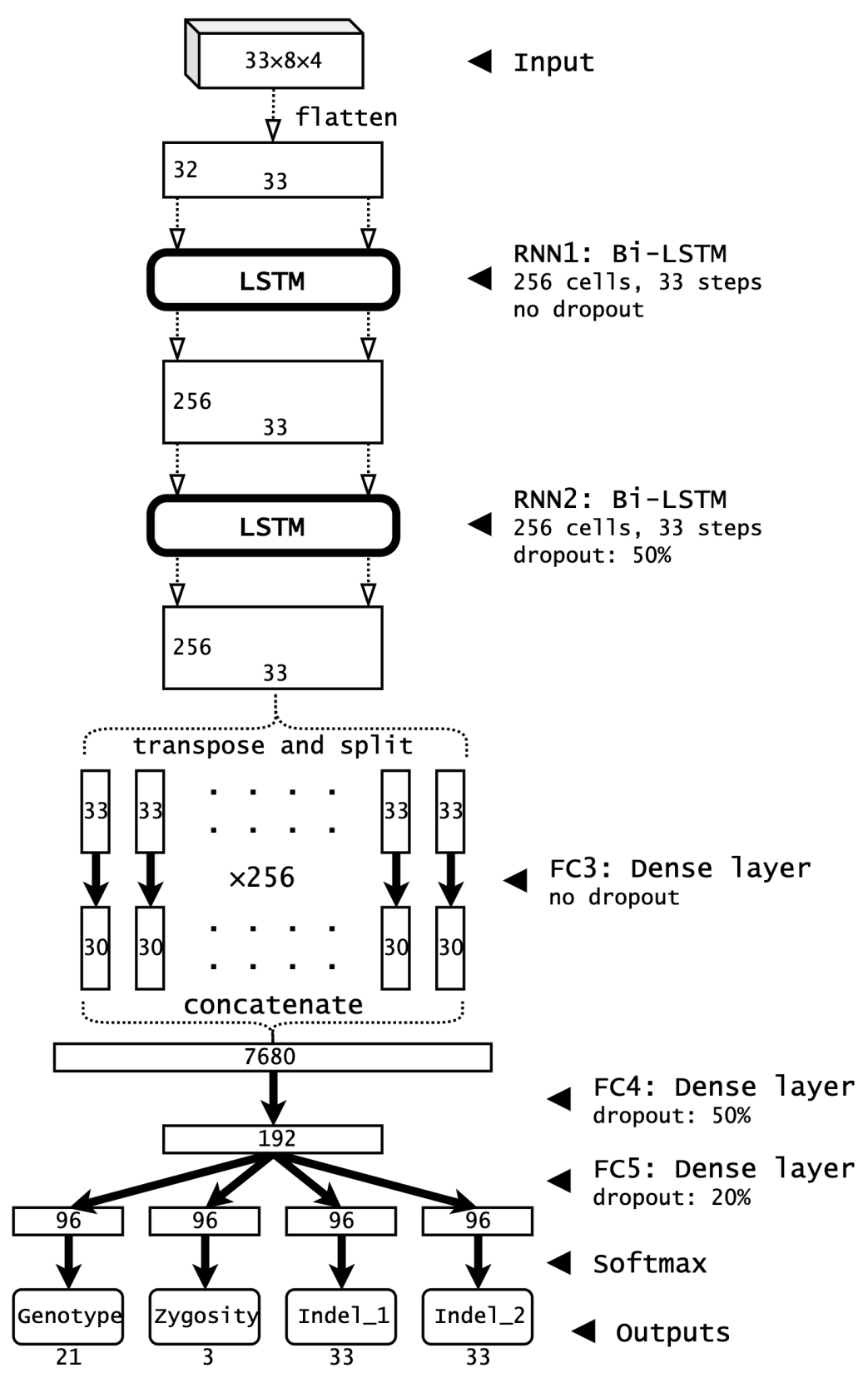

680 Figure 1. Clair network architecture and layer details. RNN: Recurrent Neural Network. FC:

681 Fully Connected layer. Bi-LSTM: Bi-directional Long Short-Term Memory layer. 

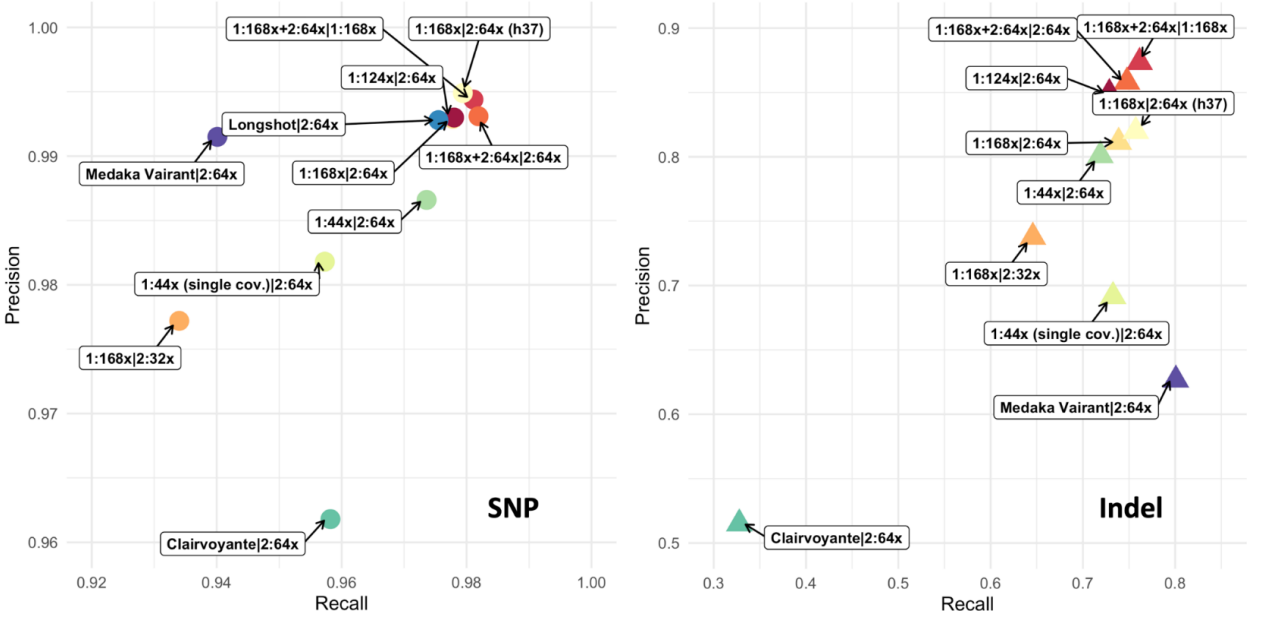

Variant type

SNP

$1: 124 x \mid 2: 64 x$

$1: 168 x+2: 64 x \mid 1: 168 x$

$1: 168 x+2: 64 x \mid 2: 64 x$

1:168x|2:32x

1:168x|2:64x 1:168x|2:64x (h37)

1:44x (single cov.)|2:64x

$1: 44 x \mid 2: 64 x$

Clairvoyante|2:64x

Longshot|2:64x

Medaka Vairant|2:64x

684 Figure 2. ONT benchmarking results. For Clair, the datasets used for model training and

685 testing are separated with a vertical bar 'I', and are written as ' $a: b x^{\prime}$, where $a$ denotes the

686 suffix of the GIAB sample ID (e.g., 1 means HG001), and $b$ denotes the coverage of the

687 dataset. Longshot calls only SNP variants, so it is not shown in the indel results. 

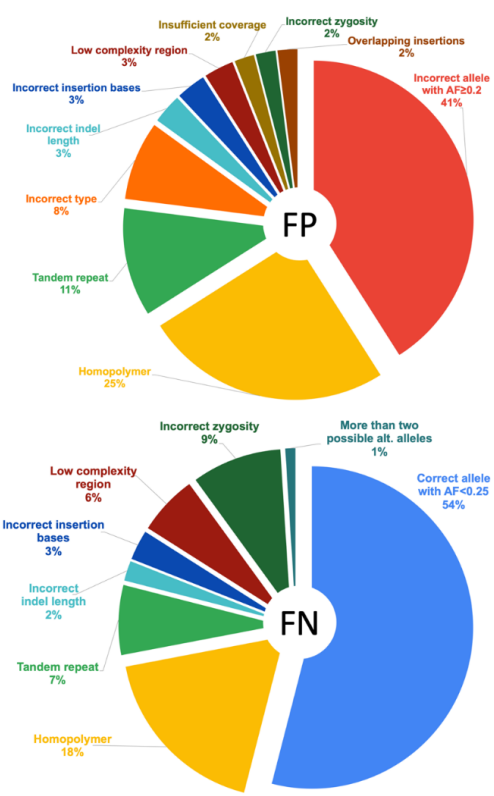
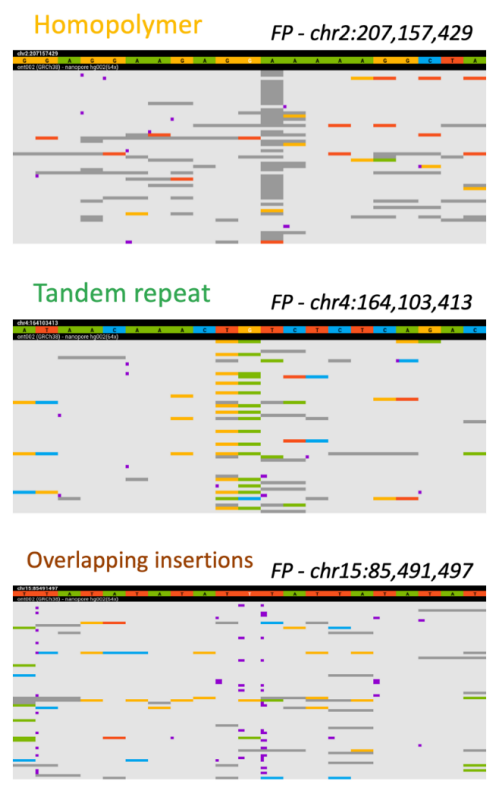
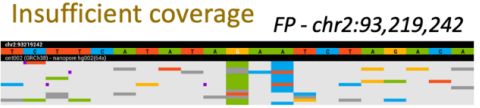

Low complexity region $\quad F N$-chr1:119,620,624

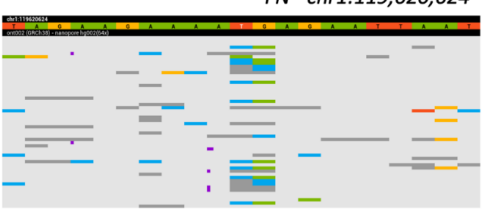

More than two

possible alt. alleles $\quad F N$ - chr6:130,007,104

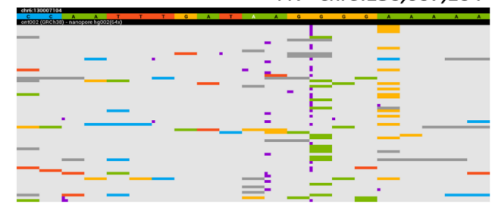

690 Figure 3. The category distribution of FPs and FNs made by Clair in the 1:168x|2:64x

691 experiment on ONT data, and six genome browser screen captures showing examples of

692 different categories. In the screen captures, bases A, C, G, and T are green, blue, yellow, and

693 red, respectively. Gaps (i.e., deletions) are dark gray. Insertions are purple dots between

694 two bases and are wider when the insertion is longer. 